\title{
Circumflex artery injury during mitral valve repair: Not well known, perhaps not so infrequent-lessons learned from a 6-case experience
}

\author{
Gonçalo F. Coutinho, MD, PhD, Filipe Leite, MD, and Manuel J. Antunes, MD, PhD, DSc
}

\author{
From the Center of Cardiothoracic Surgery, University Hospital and Faculty of Medicine, Coimbra, Portugal. \\ Received for publication March 8, 2017; revisions received April 12, 2017; accepted for publication May 7, 2017; \\ available ahead of print June 16, 2017. \\ Address for reprints: Manuel J. Antunes, MD, PhD, DSc, Cardiothoracic Surgery, University Hospitals, 3000-075 \\ Coimbra, Portugal (E-mail: antunes.cct.chuc@sapo.pt). \\ J Thorac Cardiovasc Surg 2017;154:1613-20 \\ $0022-5223 / \$ 36.00$ \\ Copyright (c) 2017 by The American Association for Thoracic Surgery \\ http://dx.doi.org/10.1016/j.jtcvs.2017.05.043
}

Mitral valve (MV) repair is the procedure of choice for the treatment of severe mitral regurgitation (MR), particularly in degenerative disease with leaflet prolapse. ${ }^{1,2}$ Annuloplasty procedures, with rings or bands, enable remodeling of the mitral annulus to adequate size and shape, enhance the coaptation area, and prevent further dilatation, and have become practically mandatory in the modern MV repair era. ${ }^{3}$ However, the vicinity of the left circumflex coronary artery (CxA) to the mitral annulus, especially near the anterior commissure, where it can run as close as $1 \mathrm{~mm}$ of the native annulus, ${ }^{4}$ places this artery at risk during MV surgery. There are scarce reports in the literature addressing this issue, which requires a comprehensive knowledge of the underlying mechanisms, prevailing factors, and therapeutic measures to minimize its devastating consequences.

We describe a series of 6 consecutive patients who had an iatrogenic CxA lesion during MV repair, which were identified from a series of more than 2500 patients who underwent MV repair from 2000 to 2016, and attempt to provide a current understanding of this entity.

\section{CASE 1}

A 25-year-old man was referred for MV surgery as a result of severe MR due to bileaflet prolapse and marked annular dilatation. Because of the patient's young age, a coronary angiogram was not performed. Intraoperative inspection showed severe Barlow's disease.

Surgery consisted of quadrangular resection of the P2 segment and implantation of 3 pairs of expanded polytetrafluoroethylene (ePTFE) (Gore-Tex, WL Gore \& Associates, Inc, Flagstaff, Ariz) artificial chordae to the prolapsing anterior segments. A size 34-mm Carpentier-Edwards Physio ring (Edwards Lifesciences, Irvine, Calif) was implanted using interrupted sutures. After removal of the aortic clamp, the electrocardiogram (ECG) showed signs of posterolateral ischemia (ST elevation), which at that time was attributed to air embolism, because it soon disappeared and

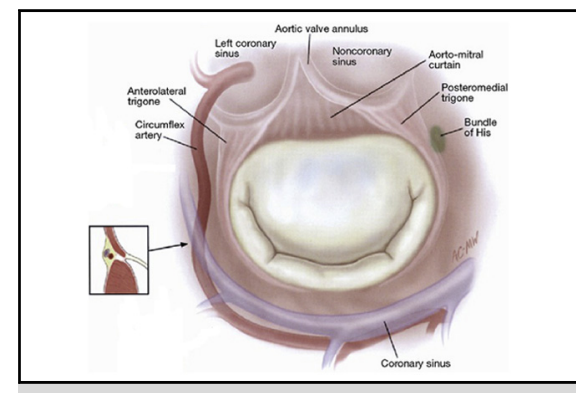

The close spacial relationship between the circumflex artery and the mitral annulus. Reprinted with permission from: Carpentier A, Adams DH, Filsoufi $F$. Carpentier's Reconstructive Valve Surgery. Philadelphia, PA: Saunders; 2010.

\section{Central Message}

Circumflex artery injury during MV surgery is most likely under-recognized and underreported. Prompt diagnosis and treatment are associated with the most favorable outcomes.

See Editorial Commentary page 1621.

cardiopulmonary bypass (CPB) was easily discontinued without inotropic support. Transesophageal echocardiography (TEE) revealed a perfectly competent valve with good leaflet coaptation.

The patient's first day in the intensive care unit (ICU) was uneventful, and the immediate ECG did not exhibit signs of ongoing ischemia. However, the ECG performed the next day revealed an inferior infarction (new-Q waves in the inferior leads), and the echocardiogram showed posteroinferior dyskinesia and poor left ventricular (LV) function without residual MR. Biochemistry data confirmed the infarction. Coronary embolism was considered the most likely cause, and the angiography performed on postoperative day 5 disclosed total occlusion of the $\mathrm{CxA}$ in a balanced circulation (Figure 1).

No additional therapeutic measures were taken since more than 24 hours had passed, the infarction was already constituted, and no evidence of persistent ischemia was observed. The patient was discharged from the hospital on postoperative day 8 , but the echocardiogram showed severe LV dysfunction, with an ejection fraction (EF) of $29 \%$. He underwent reoperation elsewhere 2 years later for a large posteroinferior aneurysm and recurrence of severe MR. 


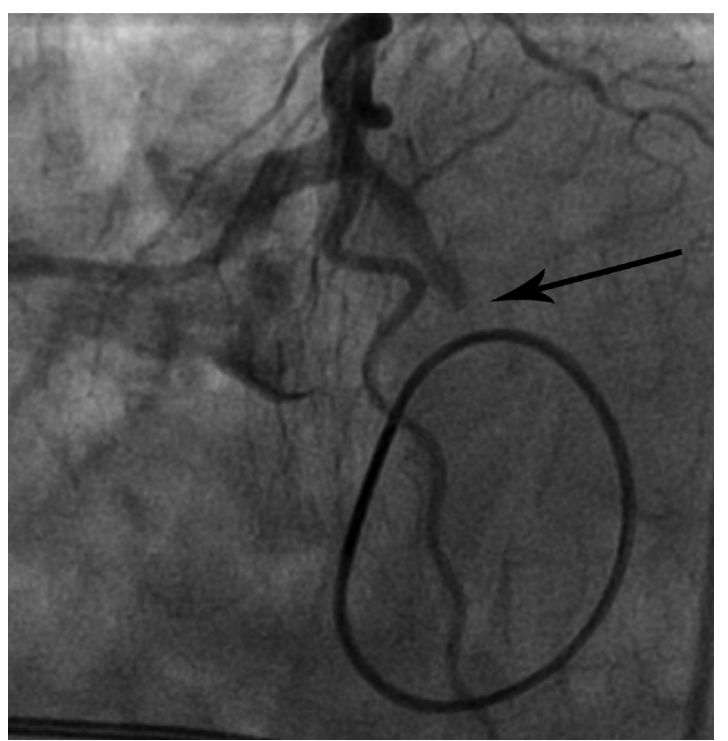

FIGURE 1. Coronary angiogram in case 1 revealing total occlusion of the circumflex artery.

\section{CASE 2}

A 66-year-old man underwent MV surgery for severe MR caused by leaflet prolapse. Preoperative study showed a normal-sized and functioning LV, severe MR, and absence of coronary artery disease, with left dominance. Intraoperative inspection of the MV evidenced thickened leaflets (myxomatous), extensive prolapse of the whole posterior leaflet ( $\mathrm{P} 1$ to $\mathrm{P} 3$ ), due to ruptured chordae, and prolapse of A2 segment.

Surgery consisted of wide resection of the $\mathrm{P} 2$ segment, with implantation of 2 pairs of ePTFE chordae to the remaining segments, in the margins of the excised area, and another 2 pairs to the A2 segment. A 34-mm Physio ring was implanted. CPB was discontinued without inotropic support. TEE showed a competent valve and moderate LV dysfunction, but no suspicion of ischemia was raised at that time.
The immediate postoperative period was complicated by low output syndrome, requiring high doses of inotropes and intra-aortic balloon pumping. Despite the absence of electric and echocardiographic signs of ongoing ischemia or infarction, emergency coronary angiography was performed 3 hours after surgery, which evidenced a critical lesion of the proximal part of the CxA (Figure 2, A). The obstruction was balloon dilated and a bare metal stent was implanted, restituting full patency of the vessel (Figure 2, $B$ ). However, the patient did not improve and required further mechanical support (centrifugal pump). Because the LV function did not recover, he underwent successful transplantation on postoperative day 10 .

\section{CASE 3}

A 48-year-old woman underwent MV repair for severe MR. Preoperative cardiac catheterization showed a dominant right coronary circulation and excluded atherosclerotic disease. Intraoperative findings revealed P2 and posteromedial commissure prolapse involving both $\mathrm{P} 3$ and $\mathrm{A} 3$ segments.

Surgery consisted of quadrangular resection of P2 segment, shortening of the posteromedial papillary muscle, and implantation of a pair of ePTFE chordae in the transition between P2 and P3 segments. A 32-mm Physio ring was implanted. The intraoperative TEE showed a normally functioning MV with no regurgitation. The patient was easily weaned from $\mathrm{CPB}$, and the surgery ended uneventfully.

Although the patient remained well in the ICU, the immediate postoperative 12-lead ECG revealed inferior and posterior ischemia associated with significant elevation of cardiac enzymes. The echocardiogram, which revealed biventricular dysfunction without MR, prompted coronary angiography, performed 18 hours after surgery, which disclosed an "elbow-like" distortion of the CxA, with
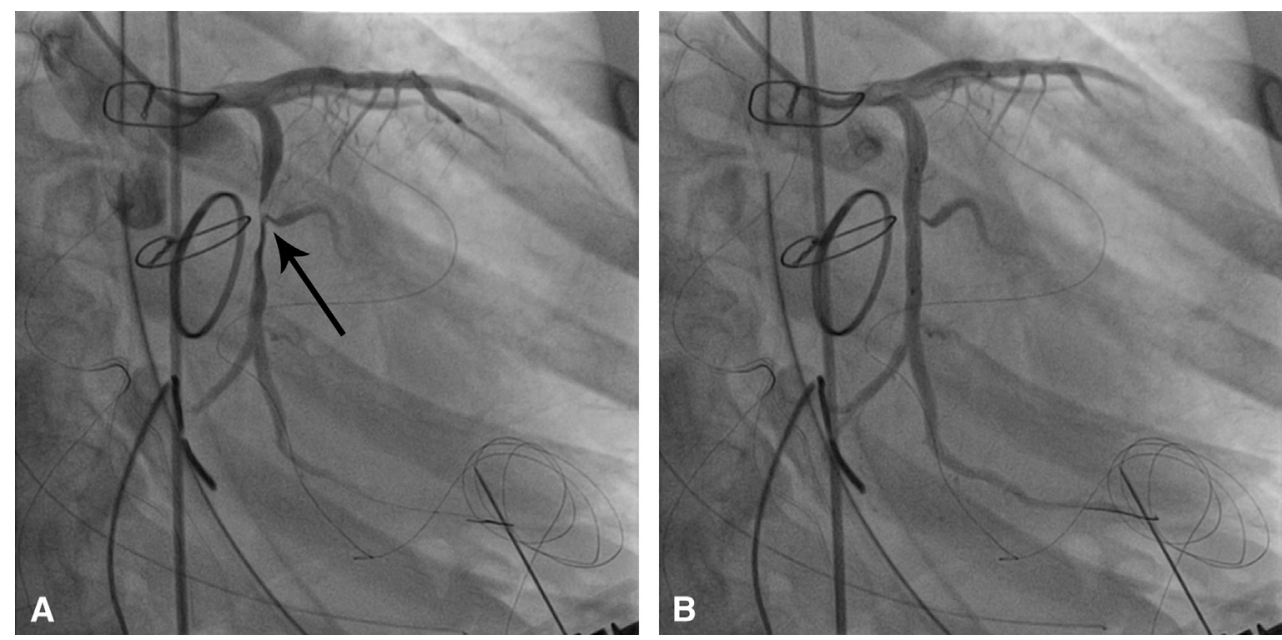

FIGURE 2. A, Immediate postoperative coronary angiogram in case 2, revealing a 90\% occlusion of the CxA (arrow). B, Result after stent implantation. 

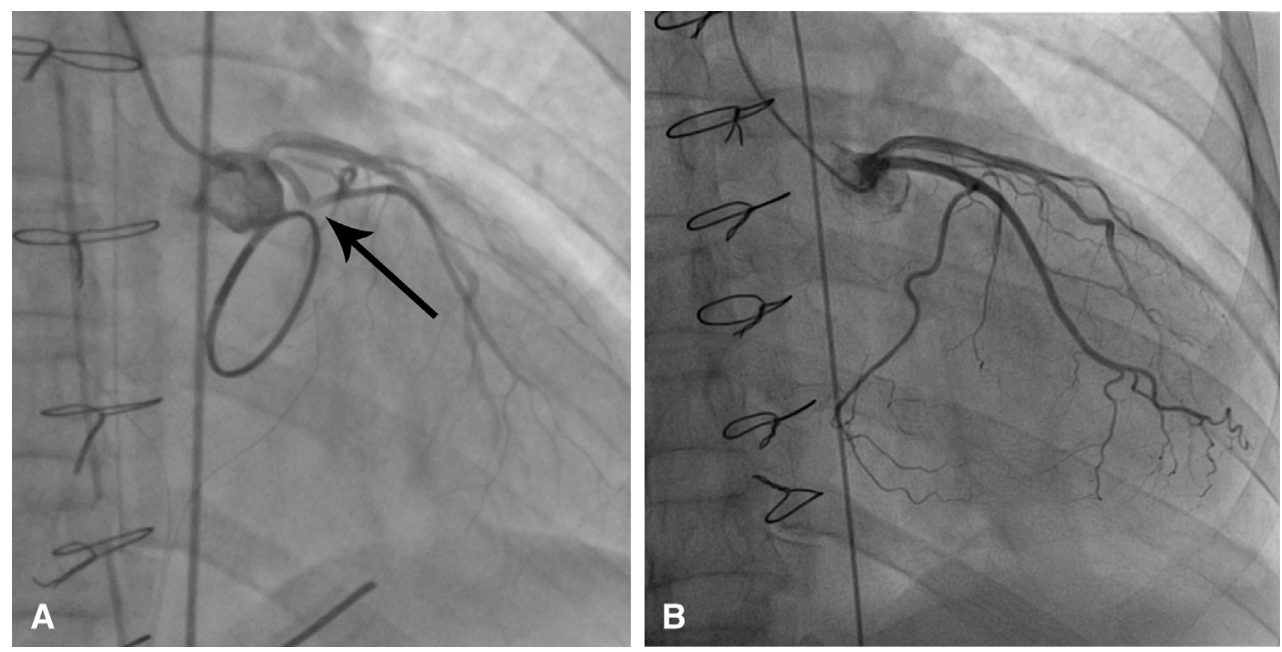

FIGURE 3. A, Coronary angiogram in case 3 revealing an "elbow-like" distortion of the CxA, with incomplete occlusion of the vessel (arrow). B, Angiogram performed 1 month after the surgery, showing no lesions.

incomplete occlusion of the vessel (Figure $3, A$ ) that was not possible to treat percutaneously. The patient was returned to the operating room for removal of the mitral ring, which was replaced by a posterior suture annuloplasty, without compromising MV competence. However, high doses of inotropes were required after surgery, and great hemodynamic instability supervened that required intra-aortic balloon pump implantation.

By postoperative day 8 , there was still moderate to severe $\mathrm{LV}$ dysfunction $(\mathrm{EF}=35 \%)$ and inferior and septal hypokinesia. Thereafter, the patient improved slowly and was discharged from hospital on the postoperative day 16 . The echocardiogram showed moderate improvement of the LV function $(\mathrm{EF}=40 \%)$. One month after surgery, a coronary angiogram was repeated revealing no lesions (Figure 3, B), but the echocardiogram was comparable to that performed at discharge.

\section{CASE 4}

An asymptomatic 49-year-old man with myxomatous mitral disease and severe MR due to anterior mitral leaflet prolapse was referred for MV repair. Preoperative coronary angiography showed a dominant left coronary circulation with no disease. Intraoperative evaluation showed A2 and A3 segment prolapse caused by ruptured and elongated native chordae, respectively. Repair consisted of implantation of 2 pairs of ePTFE chordae to A2 and 1 pair to A3, and implantation of a 32-mm Physio ring. The patient was weaned from CPB uneventfully. Intraoperative TEE revealed good leaflet coaptation and no regurgitation.

Shortly after arriving at the ICU, the patient showed ECG signs of ischemia, followed by elevation of cardiac enzymes, but there was no ventricular dysfunction and no mitral insufficiency on the echocardiogram. Nevertheless, a coronary angiogram was performed unveiling a subtotal
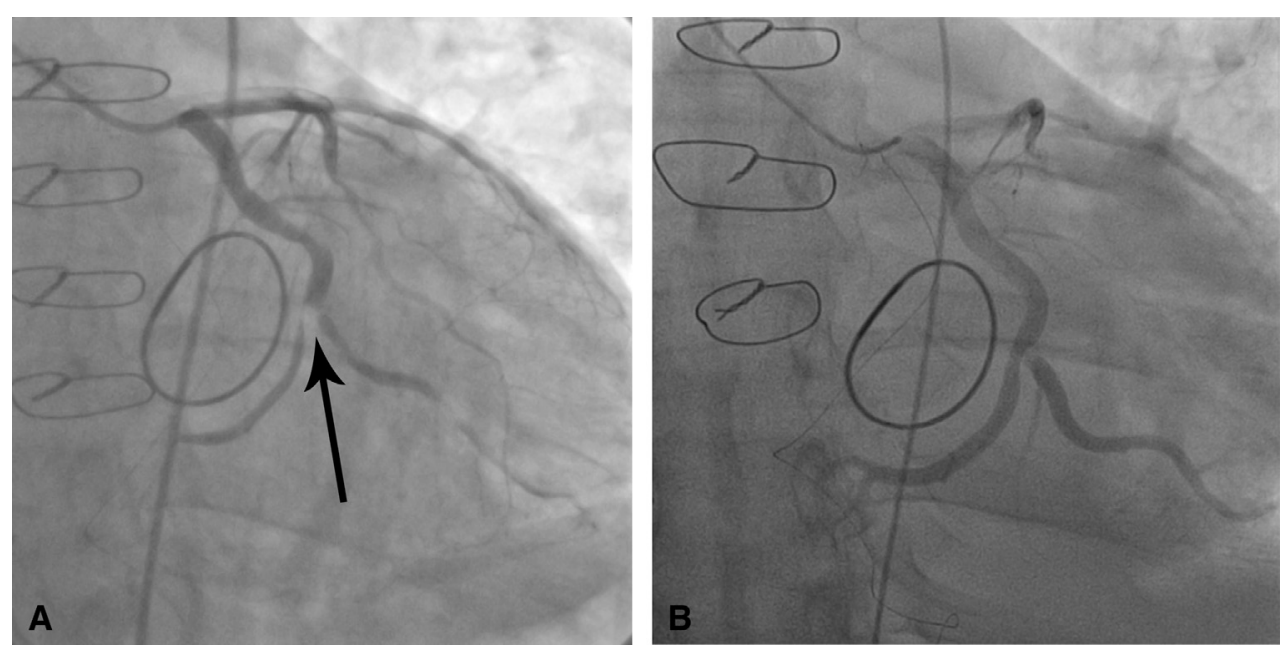

FIGURE 4. A, Immediate postoperative coronary angiogram performed in case 4, showing a subtotal occlusion of the CxA (arrow). B, Angiogram performed 1 month after discharge revealed a 50\% lesion of the CxA involving the origin of the first obtuse marginal artery. 
occlusion of the CxA (Figure 4, A). Given the past experience, the option was to reoperate on the patient and remove part of the sutures on the anterolateral region of the mitral annulus and place them more superficially, which was completed 15 hours after the end of the first surgery. The patient made an uneventful recovery.

Predischarge transthoracic echocardiogram (TTE) showed a ventricular function similar to the preoperative period, but there was mild hypokinesia of the basal interventricular septum and posterior-inferior wall, and a competent MV. One month after discharge, the coronary angiography was repeated and displayed a residual lesion of the CxA, involving the origin of an obtuse marginal branch that was treated percutaneously (Figure 4, B). The patient underwent reoperation 5 years later because of recurrence of MR and severe LV dysfunction, and the MV was replaced.

\section{CASE 5}

A 64-year-old man with severe MR caused by ruptured chordae and bileaflet prolapse (myxomatous degeneration) was referred for MV repair. Preoperative echocardiography showed good ventricular function and cardiac catheterization a balanced coronary circulation without atherosclerotic disease.

Surgery consisted of quadrangular resection of the prolapsing portion of $\mathrm{P} 2$ segment, implantation of a pair of ePTFE chordae to the A2, and implantation of a 34-mm Physio ring. When attempting to wean from $\mathrm{CPB}$, the patient exhibited signs of ischemia that raised suspicion of a CxA injury; thus, it was decided to put the patient back on bypass and extract the ring. Bypass was again discontinued with mild inotropic support and no signs of ischemia. However, intraoperative TEE revealed severely depressed LV function, with a normally functioning MV.

After the patient arrived in the ICU, the ECG showed signs of ongoing myocardial ischemia, which led to urgent coronary angiography. A relevant lesion $(50 \%-75 \%)$ was detected in the distal portion of the CxA that was treated by percutaneous ballooning 3 hours after the end of surgery (Figure 5, $A$ and $B$ ). The remaining hospital stay progressed uneventfully, and the patient was discharged home on postoperative day 8 . The predischarge echocardiogram showed hypokinesia of the interventricular septum and posterior wall, mild depression of the ventricular function $(\mathrm{EF}=50 \%)$, and no residual MR.

\section{CASE 6}

A 62-year-old man was referred for severe MR, due to posterior leaflet prolapse (P2), marked annular dilatation, and preserved $\mathrm{LV}$ function $(\mathrm{EF}=61 \%)$. Preoperative cardiac catheterization showed a dominant left coronary circulation and no atherosclerotic disease. Intraoperative findings revealed myxomatous involvement of the posterior leaflet, particularly of P2, and elongated chordae. Surgery consisted of implantation of 2 pairs of ePTFE chordae from P2 to each papillary muscle. A 32-mm Physio ring was implanted. Intraoperative TEE showed a competent MV. The patient was easily weaned from CPB, without inotropes, but in complete atrioventricular block, and the surgery ended uneventfully.

Sinus rhythm resumed spontaneously after 2 hours, when an ECG revealed elevation of the ST segment in the inferior derivations (DII, DIII, aVF) and depression of ST segment in the anterolateral leads (V4-V6). Troponin I was elevated, and the TTE showed hypokinesia of the inferior and posterior LV walls and depressed global LV function. The patient was immediately taken to the catheter laboratory. The coronary angiogram revealed complete section of the proximal CxA (Figure 6, A), which was impossible to cross with a guidewire. The patient was promptly taken to the operating room ( 8 hours after the initial surgery), the ring was retrieved, a partial suture annuloplasty limited to the posteromedial half of the annulus was performed, and a saphenous venous graft was implanted into the first obtuse marginal. Weaning from CPB was done with mild inotropic support.

The TTE performed the next day demonstrated mild to moderate LV dysfunction (EF, $40 \%-45 \%$ ), maintaining inferior and posterior hypokinesia, without MR. From postoperative day 2 , there was a progressive deterioration of the clinical status, but the TTE did not show changes in comparison with the previous one $(\mathrm{EF}=50 \%)$. The overall picture improved from postoperative day 8 onward, but the TTE performed at this time revealed severe LV dysfunction ( $\mathrm{EF}=25 \%-30 \%$ ), marked posterior and inferior hypokinesia, and mild and minimal tricuspid and MR. The patient remained in the ICU until day 20 and received another coronary angiogram that showed a patent $\mathrm{CxA}$, as well as the saphenous vein graft (Figure 6,B), with competitive blood flow. He was discharged on postoperative day 40 , after radionuclide angiography confirmed severe LV dysfunction $(\mathrm{EF}=29 \%)$.

\section{DISCUSSION}

Because of its close proximity to the MV annulus, the CxA artery is susceptible to injury during MV surgery. This complication has long been identified, ${ }^{5}$ but scarce reports in the literature have hindered the recognition of its true incidence and a comprehensive knowledge of its clinical presentation and diagnosis. ${ }^{4,6-12}$ Thus, there is lack of consensus concerning the best strategy to deal with this potentially lethal situation.

As far as we know, CxA injury occurred in less than $0.3 \%$ of our patients submitted to MV repair, but the incidence may be as high as $1.8 \% .{ }^{13}$ These rates may be underestimated for several reasons: The situation can evolve 

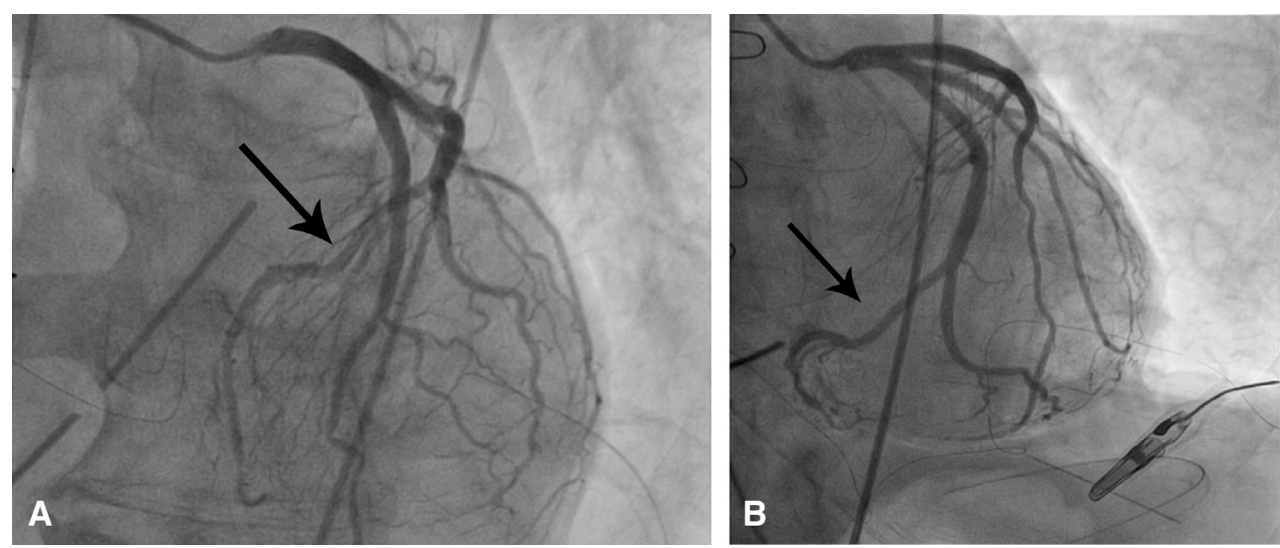

FIGURE 5. A, Coronary angiogram performed in case 5, showing a 50\% to $75 \%$ lesion of the distal CxA (arrow). B, Result after balloon dilation (arrow).

with undetected silent ischemia; some cases can be confounded by other causes of ischemia (air or particle embolism) or unrelated low output syndrome; measurement of cardiac enzymes is not routine after isolated MV surgery; and there is inherent reluctance to publish negative reports. According to the 2010 Society of Thoracic Surgeons database, the risk of perioperative myocardial infarction, which may be due to circumflex artery injury, is $1.7 \%$ for isolated MV replacement and $2.2 \%$ for MV repair, and the advent of minimally invasive MV surgery did not seem to decrease the risk of this complication and several cases have been reported using this technical approach.

Understanding of the coronary anatomy and its variability, along with the perception of the wide variation of the clinical manifestations of CxA injury and the various therapeutic strategies, is of paramount importance to delineate a standard approach to this complication.

\section{Mechanisms of Circumflex Artery Injury}

The CxA courses along the left atrioventricular groove and in $85 \%$ to $95 \%$ of patients ends near the obtuse margin of the LV, but in $5 \%$ to $15 \%$ of patients it continues to the crux of the heart to originate the posterior descending artery (dominant left coronary circulation). ${ }^{14}$ In this situation, it runs next to the anterolateral commissure and corresponding half of the posterior MV annulus (P1 segment).

The distance between the two (CxA - annulus) could be as short as $1 \mathrm{~mm}$, and several studies have identified variations according to the coronary circulation dominance. Virmani and colleagues ${ }^{15}$ were the first to report the anatomic relationship between coronary dominance and $\mathrm{CxA}$ injury. They concluded that the greater proximity to the annulus in patients with left/balanced dominance compared with the most common right dominance $(4.1 / 5.5 \mathrm{~mm}$ vs $8.4 \mathrm{~mm}$ ) could act as a potential risk factor for the injury. Others reached the same conclusion. ${ }^{16,17}$ By contrast,
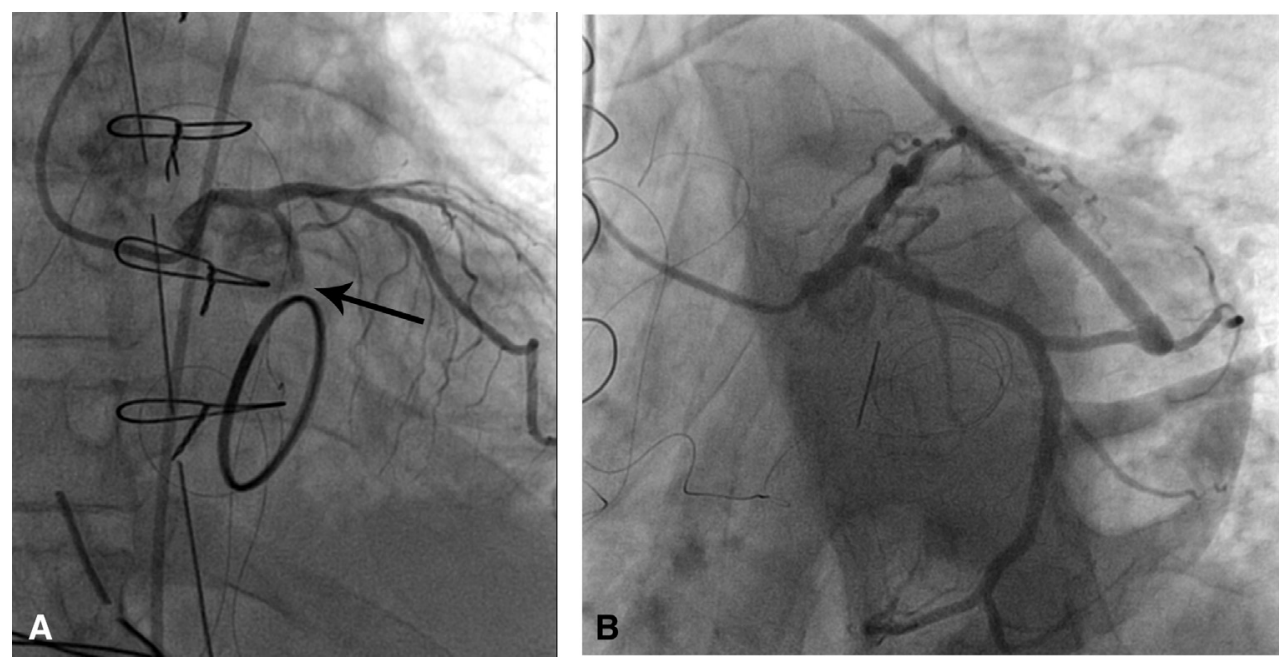

FIGURE 6. A, Immediate postoperative coronary angiogram performed in case 6, showing total occlusion of the CxA (arrow). B, Angiogram performed during hospitalization, revealing full patency of the $\mathrm{CxA}$ and the saphenous venous graft. 
Pessa and colleagues ${ }^{4}$ found no significant differences in the distance CXA - annulus between the different types of coronary dominance, but their study evaluated only 2 hearts with left dominant circulation, which raises some concern about the conclusions. Effectively, approximately $90 \%$ of the published cases, and 5 of our 6 cases, of iatrogenic injury of the CxA during MV surgery occurred in patients with left dominant or codominant circulation. ${ }^{18}$

There are several possible mechanisms of CxA injury: direct laceration of the vessel producing localized hemorrhage, complete encircling by a suture, and distortion due to tissue retraction (eg, "large bites" in the annulus during ring implantation, extensive quadrangular resection), causing dynamic or fixed occlusion. Other causal mechanisms are spasm and external compression by the ring. Most common appears to be tissue distortion and kinking of the artery, but sometimes there is no clear explanation for the injury. ${ }^{5,13,15,19-22}$ Careful placement of the sutures, 1 or $1.5 \mathrm{~mm}$ behind the hinge of the annulus, and the needle oriented toward the ventricle will probably help to avoid this complication, but crimping of the annulus, rather than the sutures themselves may be the causative mechanism.

\section{Clinical Manifestations and Diagnostic Workout}

Diagnosis of CxA injury can be more cumbersome than one imagines because the clinical presentation is highly variable and may even be absent. It can be suspected intraoperatively, early after surgery, in the ICU, and a few days or even months after the procedure. ${ }^{18}$

Difficulty to wean from CPB is probably the main presentation mode, from electrical disturbances, such as repeated episodes of ventricular fibrillation, sustained or nonsustained ventricular tachycardia, and supraventricular arrhythmias to impaired LV contractility associated with severe hypotension and high filling pressures. However, myocardial stunning may result from long CPB or aortic clamping times and LV dilatation during multiple MV tests.

Ischemic ST changes, especially ST elevation in the inferior wall derivations, should raise suspicion of CxA injury and are frequently encountered in the operative period, but also can be caused by air and particle embolism, and coronary spasm. Besides, they may not be easily identifiable in the usual 3-lead ECG. The degree of coronary obstruction (partial vs total), location of the lesion (proximal vs distal), pattern (fixed or dynamic), and the amount of myocardium at risk play an important role in the clinical presentation and constitute the reasons for the great variability.

Intraoperative TEE is now mandatory in centers performing MV repair, and its importance goes well beyond evaluation of the repair. It is crucial for assessment of proper de-airing of the heart, LV and right ventricle function, and detection of regional wall motion abnormalities (RWMAs).
Decreased cardiac contractility and RWMA are relevant findings in this complication. In 2 of our patients, intraoperative TEE detected moderate-severe LV dysfunction, previously nonexistent. ${ }^{23}$ The appearance of RWMA instead of a globally depressed ventricle should raise strong suspicion of CxA compromise, enabling rapid intervention during the same operative session, as we did in one of our cases. Otherwise, Ender and colleagues ${ }^{24}$ described an echocardiographic method to visualize the course and flow of the CxA, as well as to classify the coronary dominance pattern in patients undergoing MV surgery. Through color Dopplersignal, they could detect the course of the artery in $86 \%$ to $99 \%$ of patients and were able to identify CxA obstruction during surgery in 3 (Figure 7).

After arrival in the ICU, the diagnosis usually is suspected by ischemic changes in the 12-lead ECG or by hemodynamic instability or low output. In our series, all patients but 1 had ST-segment elevation, the majority required inotropes, and 2 needed mechanical support. Enzyme biomarkers and repeated echocardiographic examination can further help to obtain a diagnosis.

Coronary catheterization is the gold standard for anatomic diagnosis, and the threshold for its execution should be low. Also, it has the potential advantage of immediate correction of the lesion through angioplasty (percutaneous coronary intervention [PCI]). More recently, other diagnostic modalities have been suggested, such as intravascular ultrasound and multidetector computed tomography angiography. ${ }^{25,26}$ The latter may be an excellent preoperative adjunct to predict CxA injury, because it can be easily performed and is readily available, also providing detailed information of the surrounding structures.

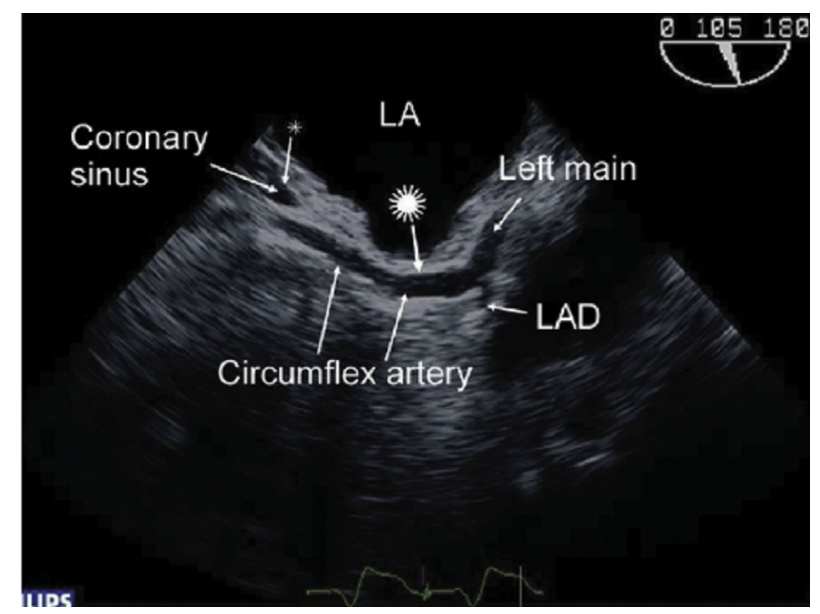

FIGURE 7. Color Doppler of the CxA. Large asterisk shows strong echodense wall of the CxA, and small asterisk shows weak echodense wall of the coronary sinus. $L A$, Left atrium; $L A D$, left anterior descending artery; left main, left main coronary artery. From Ender et al. ${ }^{24}$ 


\section{Treatment Strategy and Results}

The paucity of reported cases does not permit a definition of the best treatment modality (surgery vs PCI). Surgical treatment may be of 2 types: MV reintervention and coronary artery bypass grafting. The latter may be required to treat complete obstructions (vessel ligation). It is also more adequate when the $\mathrm{CxA}$ injury is suspected intraoperatively and there is a known large territory at risk supplied by an obtuse marginal artery with a good distal vascular bed. Alternatively, reexploring the MV and partially or completely removing the ring or band may be preferred. There are 3 other possible actions to take: relocate the sutures toward the leaflets, pass them more superficially near the anterolateral commissure and the P1 and P2 segments (usually 3-4 sutures), or simply remove the presumable culprit sutures ( 2 or 3 in that position).

The size of ring probably plays a significant role in the development of this complication. Use of small rings in severe dilated annuli and large myxomatous valves is an important risk factor. Thus, if the native annulus is severely dilated and the implanted ring was excessively downsized, it should be replaced by a larger one. Avoiding ring reimplantation if the valve stays competent or performing a posterior suture annuloplasty also may be an option. Finally, if the valve becomes incompetent after ring explantation, valve replacement may be required.

The alternative to surgery is PCI (balloon or stent) and is particularly useful when the patient is stable or when the diagnosis is made after leaving the operating room or in the catheter laboratory. If the vessel is only partially occluded, it is generally a straightforward procedure with high success rates. Nonetheless, Hiltrop and colleagues ${ }^{18}$ reported a slightly higher success rate for surgical treatment by comparison with PCI ( $87 \%$ vs $81 \%)$.

With the exception of the first patient, when our awareness of this complication was less well developed, the approach in all our other cases was accordant with those described in the literature, ranging from coronary revascularization (PCI or coronary artery bypass grafting) to ring explantation or suture repositioning. However, the results were rather disappointing, even in cases with incomplete coronary occlusion. Although all patients overcame the acute phase and are alive, the majority remained with some degree of LV dysfunction and 1 required heart transplantation, whereas 2 required late reoperation and valve replacement. But the most favorable course occurred in those patients in whom diagnosis was made precociously (intraoperatively or immediately on arrival in the ICU), enabling a rapid response.

\section{CONCLUSIONS}

Circumflex artery injury has long been recognized as a complication after MV surgery. It is most likely under-recognized and under-reported because of subclinical presentation and other confounding causes of ischemia or impaired LV contractility.

A high level of suspicion is essential for the early detection of the problem and rapid restoration of patency of the artery before myocardial infarction supervenes. Left coronary dominance or an anomalous CxA is a factor of risk and should increase suspicion. Some technical aspects should be considered to prevent this complication, such as performing large quadrangular leaflet resections and avoiding downsizing marked dilated annuli and using large and deep suture "bites," particularly at the anterior commissure level and P1 segment and adjacent P2.

The best strategy to treat CxA injury is yet to be defined, but promptness of diagnosis and treatment seem to be more important than the procedure itself (surgery vs PCI). There should be a low threshold for performing coronary angiography at the slightest suspicion, and it should be ideally performed in a hybrid room. Patients with an intraoperative diagnosis and who are hemodynamically unstable favor surgery, whereas patients with a postoperative diagnosis (ICU) and who are stable usually are better treated by PCI. Finally, a wide multicenter registry of this entity would help to know its true prevalence and to determine a risk profile that facilitates implementation of measures to avoid this dire complication.

\section{Conflict of Interest Statement}

Authors have nothing to disclose with regard to commercial support.

\section{References}

1. Vahanian A, Alfieri O, Andreotti F, Antunes MJ, Baron-Esquivias G, Baumgartner H, et al. Guidelines on the management of valvular heart disease (version 2012): The Joint Task Force on the Management of Valvular Heart Disease of the European Society of Cardiology (ESC) and the European Association for Cardio-Thoracic Surgery (EACTS). Eur Heart J. 2012;33:2451-96.

2. Nishimura RA, Otto CM, Bonow RO, Carabello BA, Erwin JP III, Fleisher LA, et al. 2014 AHA/ACC guidelines for the management of patients with valvular heart disease: a report of the American College of Cardiology/American Heart Association Task Force on Practice Guidelines. J Am Coll Cardiol. 2014;63 e57-185.

3. Carpentier AF, Lessana A, Relland JY, Belli E, Mihaileanu S, Berrebi AJ, et al. The "physio-ring": an advanced concept in mitral valve annuloplasty. Ann Thorac Surg. 1995;60:1177-85.

4. Pessa CJN, Gomes WJ, Catani R, Prates JC, Buffolo E. Anatomical relationship between the posterior mitral valve annulus and the coronary arteries. Implications to operative treatment. Rev Bras Cir Cardiovasc. 2004;19:372-7.

5. Danielson GK, Cooper E, Tweeddale DN. Circumflex coronary artery injury during mitral valve replacement. Ann Thorac Surg. 1967;4:53-9.

6. Aubert S, Barthélémy O, Landi M, Acar C. Circumflex coronary artery injury following mitral annuloplasty treated by emergency angioplasty. Eur J Cardiothorac Surg. 2008;34:922-4.

7. Grande AM, Fiore A, Massetti M, Viganò M. Iatrogenic circumflex coronary lesion in mitral valve surgery: case report and review of the literature. Tex Heart Inst J. 2008;35:179-83.

8. Somekh NN, Haider A, Makaryus AN, Katz S, Bello S, Hartman A. Left circumflex coronary artery occlusion after mitral valve annuloplasty: "a stitch in time" Tex Heart Inst J. 2012;39:104-7. 
9. Ziadi J, Mleyhi S, Denguir R, Khayati A. Iatrogenic occlusion of the circumflex artery and left ventricle pseudoaneurysm after mitral annuloplasty. J Cardiol Cases. 2014;9:104-5.

10. Acar C. Injury to the circumflex coronary artery following mitral valve repair. Eur J Cardiothoracic Surg. 2007;31:740.

11. Banayan J, Dhawan R, Vernick WJ, McCarthy PM. Iatrogenic circumflex artery injury during minimally invasive mitral valve surgery. J Cardiothorac Vasc Anesth. 2012;26:512-9.

12. Gomes WJ. Injury to the circumflex coronary artery following mitral valve repair: a rather opposite strategy. Eur J Cardiothorac Surg. 2008;33:949.

13. Aybek T, Risteski P, Miskovic A, Simon A, Dogan S, Abdel-Rahman U, et al. Seven years' experience with suture annuloplasty for mitral valve repair. J Thorac Cardiovasc Surg. 2006;131:99-106.

14. Cohn LH. Cardiac Surgery in the Adult. 4th ed. NY: McGraw-Hill; 2012.

15. Virmani R, Chun P, Parker J, McAllister HA Jr. Suture obliteration of the circumflex coronary artery in three patients undergoing mitral valve operation. Role of left dominant or codominant coronary artery. J Thorac Cardiovasc Surg. 1982; 84:773-8.

16. Cornu E, Lacroix P, Christides C, Laskar M. Coronary artery damage during mitral valve replacement. J Cardiovasc Surg. 1995;36:261-4.

17. Kaklikkaya I, Yeginoglu G. Damage to coronary arteries during mitral valve surgery. Heart Surg Forum. 2003;6:E138-42.

18. Hiltrop N, Bennett J, Desmet W. Circumflex coronary artery injury after mitral valve surgery: a report of four cases and comprehensive review of the literature. Catheter Cardiovasc Interv. February 19, 2016 [Epub ahead of print].
19. Nakajima H, Ikari Y, Kigawa I. Rapid diagnosis and management of intraoperative myocardial infarction during valvular surgery: using intraoperative transesophageal echocardiography followed by emergency coronary artery bypass grafting without coronary angiography. Echocardiography. 2005;22:834-8.

20. Meursing DF, Boonswang NA, Dobrilovic N, Wait MA. Perioperative myocardial infarction secondary to dynamic circumflex coronary artery occlusion after mitral valve repair. Texas Heart Inst J. 2006;33:85-7.

21. Mantilla R, Legarra JJ, Pradas G, Bravo M, Sanmartín M, Goicolea J. Percutaneous coronary intervention for iatrogenic occlusion of the circumflex artery after mitral annuloplasty. Rev Esp Cardiol (Engl Ed). 2004;57:702-4.

22. Tavilla G, Pacini D. Damage to the circumflex coronary artery during mitral valve repair with sliding leaflet technique. Ann Thorac Surg. 1998;66:2091-3.

23. Wykrzykowska J, Cohen D, Zimetabum P. Mitral annuloplasty causing left circumflex injury and infarction: novel use of intravascular ultrasound to diagnose suture injury. J Invasive Cardiol. 2006;18:505-8.

24. Ender J, Selbach M, Borger M, Krohmer E, Falk V, Kaisers U, et al. Echocardiographic identification of iatrogenic injury of the circumflex artery during minimally invasive mitral valve repair. Ann Thorac Surg. 2010;89:1866-72.

25. Hong YJ, Ahn Y, Jeong MH. Role of intravascular ultrasound in patients with acute myocardial infarction. Korean Circ J. 2015;45:259-65.

26. Boussel L, Ribagnac M, Bonnefoy E, Staat P, Elicker BM, Revel D, et al. Assessment of acute myocardial infarction using MDCT after percutaneous coronary intervention: comparison with MRI. AJR Am J Roentgenol. 2008; 191:441-7. 